\title{
Estimation de la charge de pollution bactériologique des eaux des deux affluents (Mikkés et Mellah) et son impact sur la qualité microbiologique des eaux du barrage Sidi Chahed (Maroc).
}

\author{
Mehanned. $S^{1 *}$, Chahlaoui. ${ }^{1}$, Zaid. $A^{1}$, Chahboune. ${ }^{1}{ }^{1}$, Dehbi. $A^{2}$ \\ ${ }^{1}$ Equipe de Gestion et Valorisation des Ressources naturelles, laboratoire d'environnement et santé, \\ Département de Biologie, Faculté des Sciences, Université Moulay Ismail. BP 11201 Zitoune Meknès Maroc. \\ ${ }^{2}$ Université Moulay Ismail, Faculté des Sciences, Département de Chimie, Equipe Chimie Analytique et \\ Environnement, B.P. 11201, Zitoune, Meknès.
}

\begin{abstract}
Résume : - La présente étude avait pour objectif d'évaluer la qualité bactériologique des eaux de la retenue de barrage Sidi Chahed et ses affluents (Oueds Mellah et Mikkés). Les sources de pollution des eaux issues de ces retenues ont été identifiées et leur qualité a été appréciée par des analyses bactériologiques entre Décembre 2012et Novembre 2013. Les principaux indicateurs microbiens de la qualité de l'eau ont été dénombrés par la filtration sur membrane à partir des milieux de culture géloses spécifiques. Les eaux du barrage, et celles des deux oueds dans les mesures sont polluées par des coliformes fécaux dont les concentrations par $100 \mathrm{~mL}$ sont parfois supérieures à 9590 UFC $/ 100 \mathrm{~mL}$. La présence de coliformes fécaux et de streptocoques fécaux au niveau des deux Oueds et au barrage indiquent une contamination d'origine fécale humaine ou animale liée à la pratique de l'élevage dans la zone d'étude, et aussi la pression anthropique. L'interprétation des données, la corrélation existante entre les différents paramètres et la répartition des stations d'études en groupe selon le degré de contamination sont réalisées par l'analyse en composante principale (ACP). Par ailleurs, le degré de pollution diffère d'un site à l'autre selon la distance d'éloignement de la source de pollution.
\end{abstract}

Mots-clés: - Contamination, qualité, Bactériologie, ACP, barrage Sidi Chahed.

\begin{abstract}
This study aimed to assess the bacteriological water quality of the reservoir dam Sidi Chahed and these emissaries (wadisMellal and Mikess). Sources of water pollution from these reservoirs have been identified and their quality was assessed by bacteriological analysis between December 2012 and November 2013.les major microbial indicators of water quality were enumerated by membrane filtration from specific media agar culture. The dam them, those two wad is in the measurements are contaminated by fecal coliform concentrations in $100 \mathrm{~mL}$ are sometimes higher than $9590 \mathrm{CFU}$. The presence of faecal coliforms and faecal streptococci at both oeuds and Dam indicate human or animal faecal contamination related to the practice of farming in the study area, as well as anthropogenic pressure. The interpretation of the data, the correlation between the different parameters and the distribution of stations group study according to the degree of contamination are conducted by the principal component analysis (PCA). Furthermore, the degree of pollution varies from site to site depending on the distance of remoteness of the source of pollution.
\end{abstract}

Keywords: Contamination, quality, Bacteriology, PCA, Sidi Chahed dam.

\section{INTRODUCTION}

Au Maroc, pays à climat semi-aride, l'approvisionnement en eau potable et industrielle est assuré essentiellement par les eaux de surface. Depuis les années soixante, quarantaine de grands barrages ont été construits. Si la construction de ces ouvrages constituait bien une nécessité pour garantir, en toute saison, l'approvisionnement en eau indispensable à notre pays, il convenait de contrôler et de sauvegarder la qualité des eaux retenues par ces barrages. Plusieurs retenues de barrages connaissent donc des problèmes de la dégradation de leur qualité d'eau due essentiellement aux différentes sources de pollution. S'ajoute aussi le processus naturel de l'érosion du sol apportant différents éléments qui peuvent être à l'origine de l'altération de la qualité de l'eau [1]. Les ressources hydriques du Maroc sont à la fois limitées et soumises à des pressions accompagnées d'une dégradation croissante de leur qualité. Cette situation risque d'être aggravée par les changements climatiques annoncés pour le Maroc dont les conséquences pourraient avoir des effets néfastes sur le potentiel en ressources en eau, tant du point de vue quantité que qualité [2]. La pollution des eaux superficielles est très perceptible, attire de suite l'attention sur les dangers et les mesures à prendre pour la combattre. 
Les informations nécessaires à l'établissement d'une politique de sauvegarde des potentialités en eau du pays, qui se baserait sur une surveillance continue de la qualité de l'eau, cette politique seraient fournies grâce au Réseau national de Surveillance continue de la qualité de l'eau qui couvre la totalité des bassins hydrauliques du Maroc. Ce réseau a été mis en place, depuis 1987, pour assurer un suivi régulier de la qualité des ressources et la tendance de son évolution et pour définir les principales causes de pollution. Ces informations restent limitées aux résultats des analyses physico-chimiques soutenues par quelques observations faunistiques qui s'appuient sur les vertébrés aquatiques qui vivent dans les eaux superficielles des grandes rivières [3].

La pollution de l'eau due à des micro-organismes d'origine fécale est apparue très tôt dès que l'eau a été utilisée comme vecteur de l'élimination des déchets [4]. Cependant, lorsque le milieu aquatique reçoit des rejets d'origine animale ou anthropique, le nombre et le type de bactéries présentes sont capables de rendre l'eau impropre à l'utilisation humaine [5]. La présente étude consiste à déterminer la qualité bactériologique des eaux de la retenue du barrage Sidi Chahed et de ses principaux affluents (Oued Mikkés et Oued Mellah) et à évaluer la variabilité spatiale, de cette qualité bactériologique, afin de déterminer une typologie de la pollution bactérienne de ces eaux de la région étudiée par une analyse en composantes principales (A.C.P).

\section{I.1. Milieu d'étude}

\section{MATERIEL ET METHODES}

Le site du barrage sidi Chahed est situé sur l’Oued Mikkés, à environ $10 \mathrm{~km}$ de la route principale $\mathrm{N}^{\circ} 3$, reliant la ville de Fès à la ville de Sidi Kacem par une piste de $3 \mathrm{Km}$ de long (Fig 1). Son bassin versant est situé entre les villes de Fès et Meknès. Il abrite les villes d'Ifrane, d'Ain Taoujdat et plusieurs centres. Sa superficie est de l'ordre de1600 $\mathrm{km}^{2}$. Le bassin de l'Oued Mikkès est drainé par quatre affluents: l'Oued N'ja et l'Oued Atchane en rive droite, l'Oued Tizguit et l'Oued Akkous en rive gauche. Les deux premiers drainent la plaine du Sais et les deux derniers drainent le plateau de Meknès et le causse d'El Hajeb-Ifrane (Fig. 2). Le bassin versant et la cuvette du barrage sur l'Oued Mikkés et l'Oued Mellah présentent des formations triasiques salifères, les sels présents dans ces formations sont : sous forme de gypse (CaSo4 2H2O) emballé dans de l'argile; et sous forme de strates métriques à plurimétriques de sel gemme $(\mathrm{NaCl})(\mathrm{Fig} 3)$ [6].

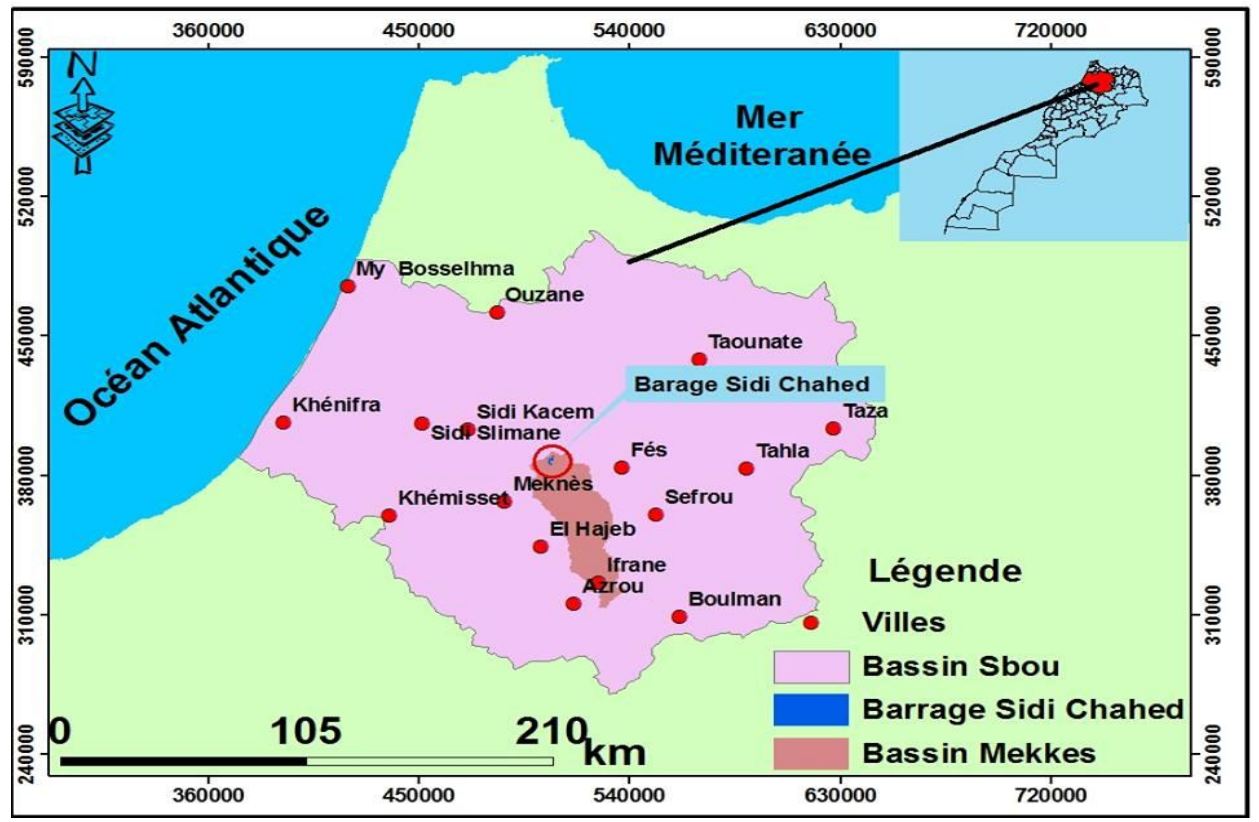

Figure 1 : Situation géographique de la zone d'étude 


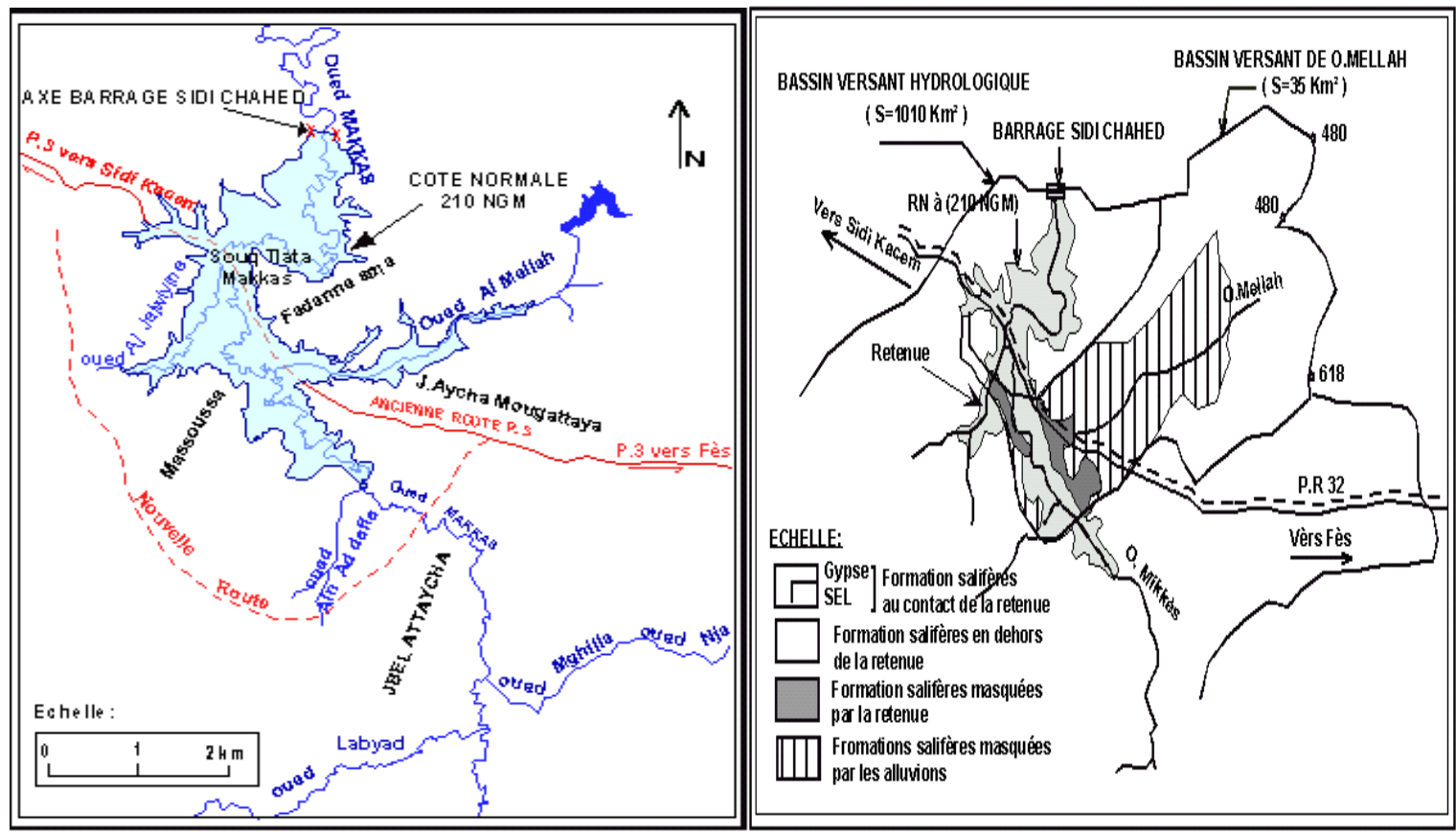

Figure 2: Carte hydrologie de barrage Sidi Chahed

Figure 3 : Zone des formations saliféres [6] de Sidi Chahed et ses affluents.

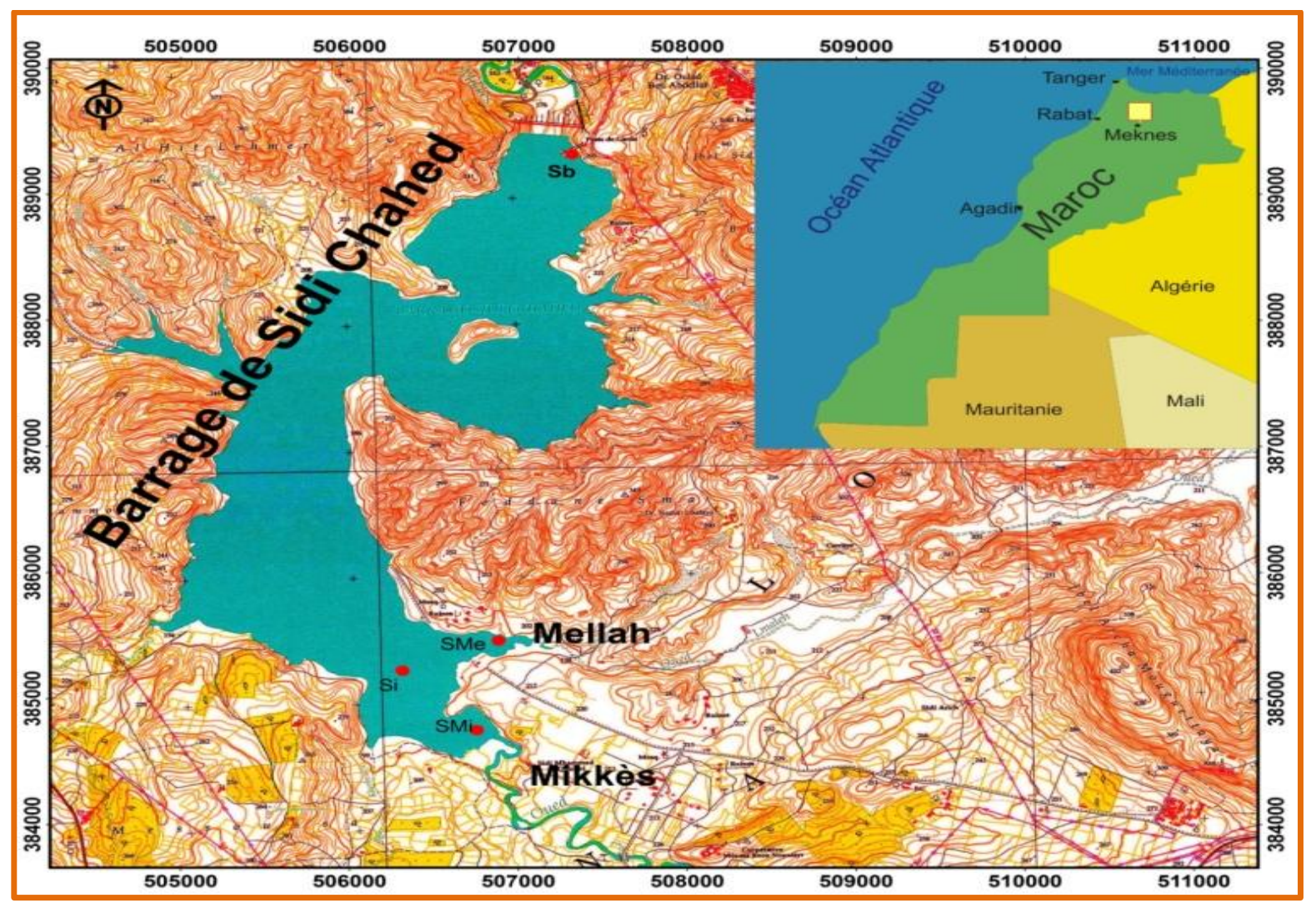

Figure 4 : Localisation des stations prospectées (SMi : Station de l'Oued Mikkés, SM Station de l'Oued Mellah, SI : Station qui rejoint les deux Oueds).

\section{I.2. Echantillonnage et Analyses.}

La période d'échantillonnage s'étale du Mois de Décembre 2012 à Novembre 2013. Les stations choisies sont : $\mathrm{Sb}$, point de prélèvement en aval, et les autres stations d'échantillonnage représentent trois points de prélèvement en amont de la retenue : les deux Oueds alimentant, l'Oued Mikkés (SMi), l'Oued Mellah(SM), et le point Si qui rejoint les deux Oueds (Fig 4). 
Les échantillons d'eau sont recueillis mensuellement dans des flacons de $500 \mathrm{ml}$ stérilisés au préalable. Le transport au laboratoire des flacons de prélèvement a été effectué dans une glacière à basse température $\left(+4^{\circ} \mathrm{C}\right)$ pour arrêter les activités métaboliques des organismes dans l'eau. Les analyses microbiologiques des eaux des stations échantillonnées sont déterminées par la méthode de filtration sur membrane; C'est la technique de concentration la plus utilisée au laboratoire. Le plus généralement, on procède à une filtration sur membranes en esters de cellulose, de porosité $0,22 \mu \mathrm{m}$ ou $0,45 \mu \mathrm{m}$, susceptibles de retenir les bactéries [7]. Les germes totaux (GT) sont dénombrés après une incubation de $24 \mathrm{~h}$ à $37^{\circ} \mathrm{C}$, par la technique d'incorporation. Cette technique, consiste à incorpore $1 \mathrm{~mL}$ d'échantillon dans une boite de pétri stérile puis faire couler le milieu PCA. Ensuite, une analyse en Composante principale (ACP) des moyens de valeurs de 12 prélèvements a été effectuée, le logiciel utilisé est la version 10 du logiciel STATISTICA.

Tableau 1 : Le dénombrement des différents germes

\begin{tabular}{|c|c|c|c|}
\hline Germes & Milieu de culture utilisé & $\begin{array}{l}\text { Température et temps } \\
\text { d'incubation }\end{array}$ & Référence \\
\hline GT & PCA & $37^{\circ} \mathrm{C}$ pendant $24 \mathrm{H}$ & $\begin{array}{c}\text { Norme AFNOR NF EN } \\
\text { ISO } 6222\end{array}$ \\
\hline $\mathrm{CT}$ & $\begin{array}{c}\text { Gélose lactosée au TTC } \\
\text { et Tergitol } 7\end{array}$ & $37^{\circ} \mathrm{C}$ pendant $24 \mathrm{H}$ & $\begin{array}{l}\text { Norme AFNOR NF EN } \\
\text { ISO 9308-1 }\end{array}$ \\
\hline $\mathrm{CF}$ & $\begin{array}{c}\text { Gélose lactosée au TTC } \\
\text { et Tergitol } 7\end{array}$ & $44^{\circ} \mathrm{C}$ pendant $24 \mathrm{H}$ & $\begin{array}{l}\text { Norme AFNOR NF EN } \\
\text { ISO 9308-1 }\end{array}$ \\
\hline SF & Slanetz et Bartley & $37^{\circ} \mathrm{C}$ pendant $24 \mathrm{H}$ & $\begin{array}{l}\text { Norme AFNOR NF ISO } \\
7899-2\end{array}$ \\
\hline ASR & $\begin{array}{l}\text { Le milieu tryptose, } \\
\text { sulfite, gélose }\end{array}$ & $37^{\circ} \mathrm{C}$ pendant $24 \mathrm{H}$ & $\begin{array}{l}\text { Norme AFNOR NF EN } \\
\text { 26461-2-ISO 6461-2 }\end{array}$ \\
\hline
\end{tabular}

II.1. Analyses statistiques

III. RESULTATS ET DISCUSSION

L'analyse bactériologique a porté sur les germes indicateurs de pollution qui regroupent les germes totaux (GT), les coliformes totaux, les coliformes fécaux, les streptocoques fécaux, et les anéarobies sulfito-réducteurs.

Tableau 2: Moyennes et extrêmes des paramètres bactériologiques des eaux des stations étudiées.

\begin{tabular}{|c|c|c|c|c|c|c|}
\hline & & GT & CT & CF & SF & ASR \\
\hline \multirow{3}{*}{ SMi } & MAX & 8000 & 9590 & 584 & 6734 & 400 \\
\hline & MOYENNE & 1740 & "4581 & 239,91 & 2016,41 & 137,33 \\
\hline & MIN & 230 & 112 & 25 & 0 & 0 \\
\hline \multirow{3}{*}{$\mathbf{S M}$} & MAX & 7320 & 9236 & 1820 & 4995 & 134 \\
\hline & MOYENNE & 1679,25 & 3429,33 & 567,16 & 577,83 & 22,916 \\
\hline & MIN & 230 & 15 & 7 & 28 & 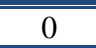 \\
\hline \multirow{3}{*}{ SI } & MAX & 2300 & 1965 & 82 & 305 & 10 \\
\hline & MOYENNE & 1061,5 & 430,08 & 33 & 104,58 & 1,66 \\
\hline & $\overline{\text { MIN }}$ & $\overline{1118}$ & 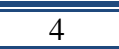 & $\overline{3}$ & $\overline{0}$ & 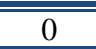 \\
\hline \multirow{3}{*}{$\mathbf{S}$} & MAX & 2783 & 6459 & 73 & 305 & 0 \\
\hline & MOYENNE & 1023,25 & 681,08 & 24,75 & 67,41 & 0 \\
\hline & MIN & 124 & 38 & 3 & 0 & 0 \\
\hline \multirow{3}{*}{ P1 } & MAX & 1996 & 8640 & 83 & 240 & 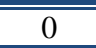 \\
\hline & \begin{tabular}{|l|} 
MOYENNE \\
\end{tabular} & 808,58 & 2517,66 & 441,58 & 67,25 & 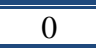 \\
\hline & $\overline{\text { MIN }}$ & 34 & 19 & 12 & 5 & 0 \\
\hline \multirow{3}{*}{$\mathbf{P 2}$} & MAX & 5400 & 1320 & 140 & 370 & 0 \\
\hline & MOYENNE & 1093,08 & 569,5 & 39 & 136,75 & $\overline{0}$ \\
\hline & MIN & 100 & 100 & 5 & 0 & 0 \\
\hline \multirow{3}{*}{$\mathbf{F}$} & MAX & 1780 & 1420 & 147 & 210 & 20 \\
\hline & MOYENNE & 675,08 & 381,66 & 62 & 85,5 & 4 \\
\hline & $\overline{\text { MIN }}$ & 259 & 28 & 12 & 15 & 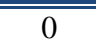 \\
\hline
\end{tabular}




\section{II.2. Germes totaux $($ GT $)$}

Ces germes regroupent tous les micro-organismes aérobies facultatifs qui apparaissent sous forme des colonies de taille et de forme différenciées [8].

Les moyennes en germes totaux des stations (SMi, SM, Si) sont respectivement $1740 \mathrm{UFC} / 1 \mathrm{ml}$; $1679,25 \mathrm{UFC} / 1 \mathrm{ml}$, et $1061,5 \mathrm{UFC} / 1 \mathrm{ml}$, donc on peut déduire de ces résultats que les stations en amont SM et SMi sont les plus contaminées en germes totaux que la station $\mathrm{Si}$ avec des valeurs les plus importants sont enregistrés au niveau de la station SMi qui constitue le réservoir des déchets de la population riveraine et des débris organiques animaux et végétaux, et des faibles valeurs en germes totaux au niveau de la station $S_{M}$ dont les teneurs en sel est très importantes induisant un stress pour les bactéries de pollution, ce principe a été démontré par plusieurs auteurs [9] [10] [11]. Sur l'ensemble de la colonne d'eau (S, P1, P2, F), les valeurs faibles en germes totaux sont enregistrées, principalement au niveau du fond de la retenue, avec des valeurs moyennes respectivement de 995,33UFC/100ml, 808,58 UFC/100 ml, 1093,08 UFC/100 ml, et 175,08 UFC/100 ml. Généralement les teneurs en germes totaux sont faibles durant la période d'étude, et les faibles valeurs en germes totaux au fond $(\mathrm{F})$ par rapport à celles trouvées aux autres niveaux de la colonne d'eau (S, P1, P2) pourraient être expliqué par des lâchers du barrage, cette situation de la contamination en germes totaux reste comparable à ceux trouvés au niveau du barrage Nahr Ibrahim au Liban [12]. D'après les résultats obtenus, on a constaté que le taux des germes totaux à $37^{\circ} \mathrm{C}$ suit un gradient amont-aval décroissant.

\section{II.3. Coliformes Totaux (CT)}

Les coliformes totaux sont considérés comme indicateurs de la qualité microbienne de l'eau parce qu'ils peuvent être indirectement associés à une pollution d'origine fécale [13]. La pertinence de ce groupe comme indicateur est aujourd'hui fortement contestée du fait que toutes les espèces inclues dans les CT ne sont pas spécifiques de la flore intestinale des animaux à sang chaud. En effet, certaines espèces sont d'origine tellurique ou aquatique et sont capables de se développer dans l'environnement aquatique [14]. Une augmentation de la contamination bactériologique de l'eau est observée au niveau de la station SMi (Oued Mikkés). Le niveau de contamination de l'eau diminue ensuite au niveau de l'intersection entre les deux oueds (Mikkés et Mellah).

La concentration moyenne des coliformes totaux de valeur $4581 \mathrm{UFC} / \mathrm{ml}$ enregistré au niveau de la station SMi avec une valeur maximale de $9590 \mathrm{UFC} / 100 \mathrm{ml}$, et minimale de 4 UFC/100 $\mathrm{ml}$ signalées au niveau de la même station, alors qu'une contamination assez élevée est enregistrée au niveau de la retenue, les valeurs extrêmes ont une valeur maximale de $8640 \mathrm{UFC} / 100 \mathrm{ml}$ marquées au premier niveau de profondeur P1, avec une valeur minimale de 19 UFC /100 ml. L'analyse a montré qu'il y a un changement est survenu concernant le taux des coliformes totaux pendant la période d'étude, où nous avons noté l'augmentation du nombre de celleci pour les échantillons des deux oueds (Mikkés et Mellah); ceci est expliqué par l'écoulement de ces deux Oueds qui alimentent le barrage, ramassent au cours de leurs trajets de nouvelles charges microbiennes. On a remarqué que la moyenne des coliformes totaux de l'eau de surface de la colonne d'eau est égale à 9590 UFC/100ml, qui est plus élevé que celle de l'eau de profonde estimé de $1420 \mathrm{UFC} / 100 \mathrm{ml}$ et cela pourraient être expliqués par des lâchers de barrage (Tableau 2).

\section{II.3. Coliformes Fécaux (CF)}

Les Coliformes fécaux thermo sont des bactéries habituelles du tube digestif de l'homme et des animaux [15]. Sa détection dans l'eau doit faire sérieusement soupçonner une contamination d'origine fécale [16].

Les valeurs les plus élevées sont portées par la station $S_{\mathrm{Mi}}$ avec une valeur maximale de $1820 \mathrm{UFC} / 100 \mathrm{ml}$ et des faibles valeurs en coliformes fécaux sont relevées dans la station $S_{i}$, avec une valeur minimale de 3UFC/100 ml, l'importance des teneurs en coliformes fécaux confirme, la contamination fécale des eaux de la station $\mathrm{S}_{\mathrm{Mi}}$, et la diminution de la contamination dans la station $\mathrm{S}_{\mathrm{i}}$ par l'effet de la dilution (éloignement des sites de contamination). D'après nos résultats d'analyses, nous avons remarqué que le taux coliformes fécaux sur l'ensemble de la colonne d'eau a fluctuée entre un minimum de 3 UFC/100 ml et un maximum de 147 UFC/100 $\mathrm{ml}$, sauf pour les échantillons prélevés au niveau des deux Oueds où nous avons remarqué une augmentation significative de taux coliformes fécaux causés le plus souvent par l'écoulement des Oueds qui alimentent le barrage ramassent au cours de leurs trajets des nouvelles charges microbiennes (la matière fécale des animaux), selon la norme marocaine N.M(03.7.200), ces eaux sont de bonne qualité pour les baignades et de bonne qualité pour l'irrigation [17].

\section{II.4. Streptocoques Fécaux (SF)}

Ces germes sont associés aux coliformes fécaux, ils sont considérés comme un bon indicateur de pollution, aussi utilisés comme indicateurs d'efficacité de traitement, car ils sont nettement plus résistants que les coliformes et autres entérobactéries pathogènes [13]. Les streptocoques fécaux présentent un maximum au 
niveau de la station SMi de 6734 UFC/100mL, probablement à cause de l'utilisation des matières fécales des animaux utilisées comme engrais dans les aires cultivées au bord de cette station, la station (SM) est caractérisée par une pollution fécale assez élevée, les streptocoques fécaux présentent un maximum au niveau de cette station de l'orde de $4995 \mathrm{UFC} / 100 \mathrm{~mL}$ avec du fait de la présence du bétail et des animaux domestiques qui ont libre accès au cours d'eau, cette contamination fécale des stations en amont reste inférieurs à celles trouvées par [18] au niveau d'Oued Boufkrane, [19] au niveau d'Oued Aggai (Moyen-Atlas). Sur l'ensemble de la colonne d'eau, on a remarqué que le moyen obtenu des streptocoques fécaux de l'eau de surface égale à 305 UFC/100mL est supérieure à celle pour l'eau de profonde qui est égale à $210 \mathrm{UFC} / 100 \mathrm{~mL}$, ces teneurs bactériennes restent inférieur à celles signalées par [20] au niveau du lac Mellah (algéris), par [21] au niveau du lac réservoir Hassan Dakhil, et par [22] au niveau du lac réservoir Hassan II. D'une manière globale la contamination en streptocoque fécaux suit un gradient décroissant amont-aval, avec des valeurs les plus importants sont portés par la station $\mathrm{S}_{\mathrm{Mi}}$ en amont, et des valeurs les moins importantes sont marqués à partir de la station $S_{i}$ et sur l'ensemble de la colonne d'eau. Selon les normes marocaines $\mathrm{NM}(03.7 .200)$, ces eaux sont de bonne qualité pour les baignades.

\section{II.5. Anaérobies Sulfito-Réducteurs (ASR)}

Les Clostridium sulfito-réducteurs sont des germes capables de sporuler et de se maintenir longtemps dans l'eau. Ils sont donc les témoins d'une pollution ancienne. Plus difficilement tués que les coliformes par les désinfectants, ils constituent aussi un bon indicateur de l'efficacité de la désinfection [23].

D'après les résultats indiqués dans le tableau 2, on a remarqué que la moyenne des Clostridium sulfito-réducteur de l'eau de surface des stations (SMi, SM, Si) est égale 137,33 UFC/20ml, 22,916 UFC/20ml, 1,66 UFC/20ml, respectivement, et sur l'ensemble de la colonne d'eau les teneurs moyennes des Anéarobies sulfito-réducteurs sont 0 UFC /100 ml de la surface à la profondeur P2, et de 4 UFC /100 ml, ce développement au niveau de fond de la colonne pourrait être expliqué par leur développement dans des milieux privés d'oxygène ou à potentiel d'oxydoréduction très bas, de ce fait, le principe de leur existence au niveau de fond de la colonne d'eau.

\section{II.6. Evaluation temporelle de rapport $\mathrm{CF} / \mathrm{SF}$}

La recherche des streptocoques fécaux envisagée par les travaux de Wilson et palmer en 1910, présente une distinction entre la pollution dominée par les rejets fécaux d'animaux et celle dominée par les rejets humains. Selon les travaux de [24], le rapport C.F/S.F, utilisé dans l'étude de la pollution des eaux est supérieur à 1 lorsque la pollution fécale est dominée par les rejets humains et inférieur à 1 dans le cas où dominent les rejets fécaux d'animaux.

Au cours de la période d'étude, la variation spatiotemporelle du rapport coliformes fécaux sur streptocoque fécaux (Fig 5), au niveau de la surface des eaux du la retenue (S), et ses affluents $S_{\mathrm{Mi}}$ (Oued Mikkés) et $\mathrm{S}_{\mathrm{M}}$ (Oued Mellah), a montré que :

En hiver : la contamination est d'origine animale à la station $\mathrm{S}_{\mathrm{Mi}}$ en décembre, et à la station $\mathrm{S}$ en décembre et janvier. D'origine humaine à la station $\mathrm{S}_{\mathrm{Mi}}$ en février et décembre, et à la station $\mathrm{S}$ en février. D'origine incertaine à la station $S_{\mathrm{Mi}}$ en février et janvier et à la station $S_{\mathrm{M}}$ en janvier.

En printemps : la contamination est d'origine animale en avril, d'origine humaine à la station $\mathrm{S}_{\mathrm{Mi}}$ en Mars et à la station $\mathrm{S}_{\mathrm{M}}$ en Mai, et d'origine incertain à la station $\mathrm{S}$ en Mars.

En été : la contamination d'origine animale à la station $\mathrm{S}_{\mathrm{Mi}}$ en juin et juillet, et à la station $\mathrm{S}$ en juin, juillet, et Aout, d'origine incertaine à la station $S_{M i}$ en Aout et $S_{M}$ en juin et Aout, et d'origine mixte à prédominance humaine à la station $S_{M}$ en juillet.

En automne : on assiste à une contamination humaine et animale à l'exception de la station $\mathrm{S}$ en octobre où la contamination est incertaine.

Il ressort de l'étude de ce rapport que la contamination humaine et animale qui prédomine en été et en automne où la contamination est très importante. Les stations en aval présentent un état de pollution d'origine animale, les stations amont $\left(\mathrm{S}_{\mathrm{M}}, \mathrm{S}_{\mathrm{Mi}}\right)$ présentent un état de pollution animale et humaine. 


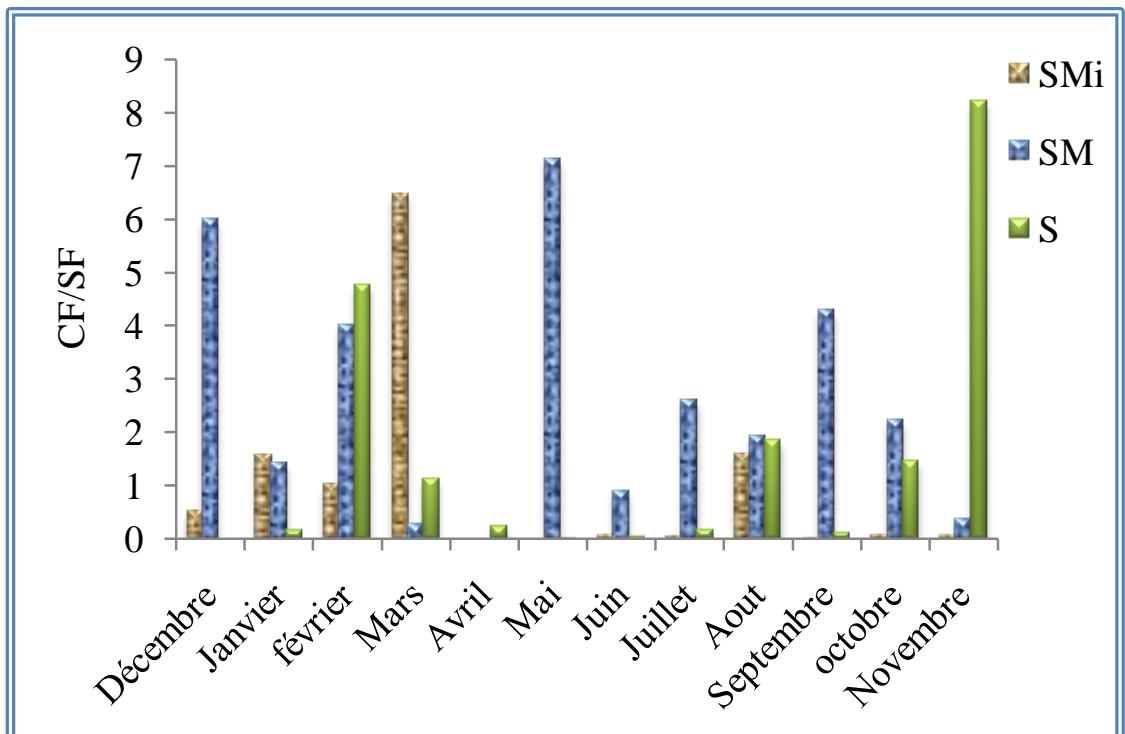

Figure 5 : Evolution spatiotemporelle des coliformes fécaux sur les streptocoques fécaux durant la période d'étude.

IV. TYPOLOGIES DES STATIONS

Tableau 3: Matrice de corrélation entre les paramètres microbiologiques (test de Person).

\begin{tabular}{|c|l|l|l|l|l|}
\hline Variable & \multicolumn{1}{|c|}{ GT } & \multicolumn{1}{c|}{ CT } & \multicolumn{1}{c|}{ CF } & \multicolumn{1}{c|}{ SF } & ASR \\
\hline GT & 1,000000 & 0,77964 & 0,73746 & 0,51312 & 0,89234 \\
\hline CT & 0,77964 & 1,000000 & 0,87746 & 0,57025 & 0,781258 \\
\hline CF & 0,73746 & 0,87746 & 1,000000 & 0,621458 & 0,770796 \\
\hline SF & 0,51312 & 0,57025 & 0,621458 & 1,000000 & 0,273619 \\
\hline ASR & 0,89234 & 0,781258 & 0,770796 & 0,273619 & 1,000000 \\
\hline
\end{tabular}

Cette étude de corrélation ne donne pas une grande information sur la distribution temporelle et spatiale de ces variables microbiologiques, c'est pour cette raison, et pour mieux cerner la diversité spatiale et temporel de ces variable, nous avons procéder à une analyse en composante principale.

L'analyse des résultats permet de constater que la majeure partie des informations est expliquée par les deux premiers axes factoriels. Les contributions des différents paramètres dans la formation des deux Premiers axes factoriels F1, F2 sont respectivement de $68,15 \%, 16,25 \%$ soit un totale de 84, $41 \%$ de l'information expliquée (Tableau 4).

Tableau 4: Contributions des trois premiers axes à la variance totale.

\begin{tabular}{|l|c|c|c|}
\hline & F1 & F2 & F3 \\
\hline Valeur propre & 2,4 & 1 & 0,7 \\
\hline Variabilité \% & $68,15 \%$ & $16,25 \%$ & $14,26 \%$ \\
\hline Cumulée\% & $68,15 \%$ & $84,41 \%$ & $98,67 \%$ \\
\hline
\end{tabular}

L'analyse en composante principale montre que les coliformes totaux, les germes totaux, les coliformes fécaux, et les Anaérobies Sulfito- réducteurs sont corrélés négativement avec l'axe F1 $(68,15 \%)$, et que les streptocoques fécaux sont corrélés positivement avec l'axe F2 (16, $25 \%$ ) (Fig 6, A).

L'évolution temporelle des eaux des sept stations étudiées durant la période d'étude, ont été évaluées par des nuages des points dispersés dans le plan factoriel F1-F2. Cette dispersion du point dépend de la modification climatique et des rejets domestiques et agricoles, toute augmentation de cette dispersion signifie une variation très importante de ces facteurs.

La fig $(6, \mathrm{~B})$ représente les stations de prélèvement dans les plans recomposés à partir des composantes 1 et 2 . De cette représentation, il ressort que les stations de prélèvement sont regroupées en trois grands groupes :

Groupe 1 : ce groupe est coordonné négativement avec l'axe F1, et formé par les relevés des stations en amont (Oued Mikkés et Oued Mellah) en printemps suivi d'été. Les eaux de ce groupe sont très riches en coliformes totaux, germes totaux, coliformes fécaux, et les anaérobies sulfito-réducteurs. 
Groupe 2 : ce groupe formé par les individus (Mjn, Mju, MSe, Moc, MNo, HNo) ce sont essentiellement des eaux de l'Oued Mikkés en été, et sont corrélés positivement avec l'axe factoriel F2. Les individus de ce groupe se distinguent par une forte charge en streptocoque fécaux.

Groupe 3 : constituent par les relevés des stations en aval (Intersection des deux Oueds et l'ensemble de la colonne d'eau) durant la période d'étude, et les stations en amont (Oued Mikkés) pendant la période pluvieuse. Ces individus sont plus proches de centre et sont faiblement chargé en coliformes fécaux, coliformes totaux, germe totaux, streptocoque fécaux, et les Anaérobies sulfito-réducteurs.
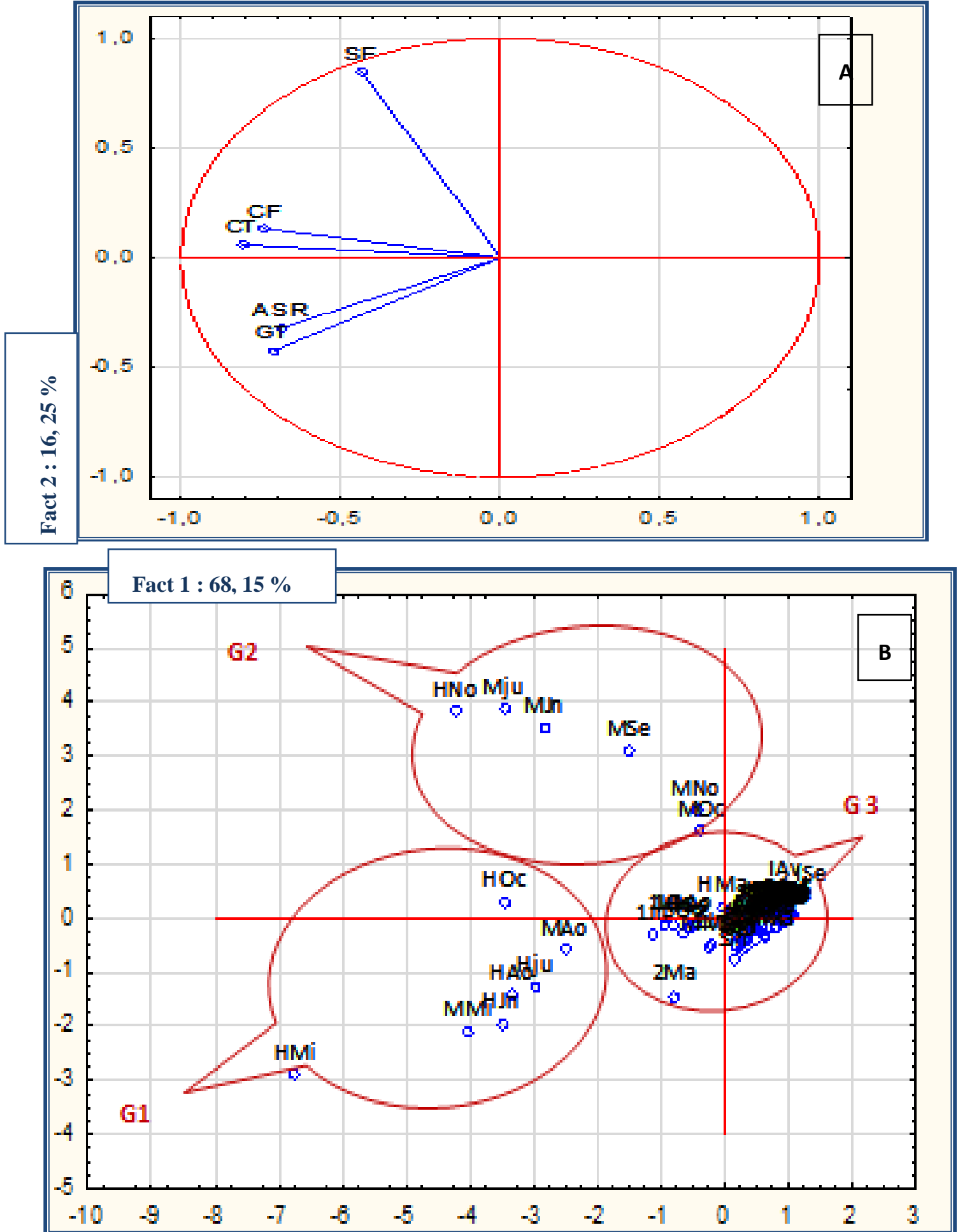

Figure 6 : Analyse en composante principale des variables bactériologiques(A)

et des individus mois-stations(B). (H: $\mathrm{S}_{\mathrm{M}}, \mathrm{M}: \mathrm{S}_{\mathrm{Mi}}$, I: SI, S: S, 1:P1, 2:P2, F: F)

(dé: Décembre, ja: Janvier, fé: Février, Ma : Mars, av : avril, Mi : Mai, Jn : juin, ju, Ao : Aout, Se : Septembre, Oc : Octobre, No : Novembre). 
Ce travail qui entre dans le cadre de l'étude de la qualité microbiologique des eaux des trois stations en amont (Mikkés, Mellah) et des stations en aval constitue par quatre niveaux de la colonne d'eau (S, P1, P2, F) de barrage sidi chahed, et le point qui rejoint les deux oueds montre que ces eaux se différent de point de vue biologique selon la saison et les stations.

Nous pouvons déduire généralement de ces résultats qu'il s'agit donc d'un gradient décroissant, ces gradients sont nettement plus prononcés en amont (Oued Mikkés, Oued Mellah) qu'en aval (le point qui rejoint les deux Oueds et sur l'ensemble de la colonne d'eau de la retenue). Malgré l'importance des bactéries indices de contaminations fécales, les Anaérobies sulfito-réducteurs n'ont pas été détectés au niveau des eaux d'oued Mellah et sur l'ensemble de la colonne d'eau à l'exception des eaux de l'Oued Mikkés et le fond de la retenue, mais cette contamination reste généralement très faible.

Il ressort de l'étude de rapport CF/SF, que la contamination humaine et animale qui prédomine en été et en automne où la contamination est très importante. Les stations en aval présentent un état de pollution d'origine animale, les stations amont $\left(\mathrm{S}_{\mathrm{M}}, \mathrm{S}_{\mathrm{Mi}}\right)$ présentent un état de pollution animale et humaine.

L'analyse en composante principale a mis en évidence l'importance du rôle de la température (étiage) et la disponibilité des matières organiques dans la distribution et l'abondance des germes tests de contaminations fécales, et a permis aussi de confirmer les résultats précédents de la contamination microbiologique saisonnière des eaux étudiées.

\section{REFERENCES}

[1] Y. EL Ghachtoul, M.A. Mhamdi, H. Gabi, Eutrophisation des eux des retenues des barrages Smir et Sehla (Maroc) : causes, conséquences et consignes de gestion, Rev.Sci. de l'Eau, 18, 2005, 75-89.

[2] J. Alibou, Impacts des changements climatiques sur les ressources en eau et les zones humides du Maroc, Table Ronde Régionale en Méditerranée Athènes, Grèce, 2002 Décembre 10-11.

[3] $\mathrm{ADH}$, Etat de la qualité des ressources en eau dans la région du sebou, Direction de la recherche et la planification de l'eau, Ministère des travaux publics et de la formation des cadres, Administration de l'hydraulique, 1993.

[4] T. Georges et J. Pierre, L'eau, patrimoine mondial commun, Belgique, (presses Universitaire de Numur, 2002).

[5] S. Hébert et S. Légaré, Suivi de la qualité des rivières et petits cours d'eau. Direction du suivi de l'état de l'environnement, ministère de l'Environnement, Québec, envirodoq No ENV-2001-0141, rapport $\mathrm{N}^{\circ} \mathrm{QE}-$ 123, 24 p. 2000

[6] ABHS, Avant projet sommaire de la réhabilitât du barrage sidi Chahed (problème de la salinité), Agence du Bassin Hydraulique de Sebou, Rapport, 2004.

[7] J. Rodier, B. Legube, N. Merlet, L'analyse de l'eau: eaux naturelles, eaux résiduaires, eau de mer. (Dunod, 9ème édition. Paris, 2009).

[8] S. Jean-noël, Bon état des eaux, Toulouse, 2008

[9] K.H. Chedad et Assobhei, Etude de la survie des bactéries de contamination fécale (coliformes fécaux) dans les eaux de la zone ostréicole de la lagune de Oualidia (Maroc), Bulletin de l'Institut Scientifique, Rabat, Section Sciences de la vie, 29,2007, 71-79.

[10] A. D. N'diaye, M. O S. A. O Kankou., A. D. Sarr et B. Lo1, Caractérisation physico - chimique des eaux usées brutes de la ville de nouakchott (mauritanie). Rev. Ivoir. Sci. Technol., 14, 2009, 97 - 109

[11] M. Bennani, H. amarouch, A. Boukanjer, H. Nader, M. Lalaoui, M. Allali, N. ET Cohen, Influnence des facteurs environnementaux sur les charges des bactéries fécales dans le littoral méditerranéen du Maroc, European Journal of Scientific Research, 71, 2012, 24-35.

[12] H. BouSaab, N. Nassif, A. G. El Samrani, R. Daoud, S. Medawar, N. Ouaïni, Suivi de la qualité bactériologique des eaux de surface (rivière nahr ibrahim, liban). Revue des sciences de l'eau / Journal of Water Science, 2, 2007, 341-352.

[13] G. Leyral, C. Ronnefoy, F. Guillet, E. Verne, Bourdais, Microbiologie et qualité des industries agroalimentaire, Paris, 2002,

[14] P. Tallon, B. Magajna, C. Lofranco, et K.T. Leung, Microbial indicators of faecal contamination in water: A current perspective. Water Air and Soil Pollution, 166, 2005, 139-166.

[15] APHA, Compendium of methods for the microbiological examination of foods, ed. Marvin L, Speck. Washington,USA, American Public Health Association, 1976.

[16] L.M. Prescott, J.P. Harley, D. Klein, J.M. Willey, L.M. Sherwood, C.J. Woolverton, Microbiologie, 3ème édition, 2010.

[17] SEEE, Etude de la stratégie nationale de l'eau, Secrétariat d'Etat chargé de l'Eau et de l'Environnement(SEEE). Rabat, 2009. 
[18] M. Larif, A. Soulaymani, M. Hnach et A. EL midaoui, Contamination spatio-temporelle d'origine hydrique de l'oued Boufekrane dans la région de Meknès-Tafilalt (Maroc), Int. J. Biol. Chem. Sci. 7, 2013, 172-184.

[19] A. Khalid, A. Nadama, K. Essafi et R. Guemmouh, Prospection de la qualité physicochimique et biologique des eaux superficielles et interstitielles de l'oued Aggai, Moyen-Atlas Sefrou, Maroc, Revue AFN Maroc $N^{\circ}$ double, 2009.

[20] W. Kherifi, H. Kherici-Bousnoubra. Evolution saisonnière de la qualité microbiologique des eaux du lac mellah (nord-est algérien), Larhyss Journal,11, 2012, 109-118.

[21] M. Ouhmidou, et A. Chahlaoui, Qualité physico-chimique des eaux du barrage hassan addakhil errachidia-maroc, ScienceLib Editions Mersenne, 5, 2013, 09-23.

[22] M. Chahboune, A. Chahlaoui, A. Zaid, Contribution a l'étude de la qualité bactériologique des eaux du barrage hassan II (province de midelt, maroc). Les technologies de laboratoire, 8, 2014.

[23] I. George et P. Servais, Sources et dynamique des coliformes dans le bassin de la Seine. Synthesis Report 1998-2001 of the scientific programme PIREN-Seine, CNRS, France, 4, 2003, 45.

[24] B. A. Kenner, H. F, Clark and P. W. Kabler, Fecal Streptococci and enumeration of streptococci in surface water, App.microbio, 9, 1961, 15-20. 